\title{
ORTHODONTIC AND IMPLANT-PROSTHETIC TREATMENT OF HYPODONTIA: A REPORT OF TWO CASES
}

\author{
Barbara Rafałowicz, Leopold Wagner \\ Department of Dental Propedeutics and Prophylaxis, Medical University of Warsaw, Warsaw, Poland
}

\begin{abstract}
Congenital absence of maxillary tooth germs in adults is a serious problem. Basic treatment includes orthodontic and/or implantoprosthetic procedures. The aim of the study is to present possible therapeutic procedures in the treatment of hypodontia of maxillary teeth in complex anatomical and biological conditions in adult patients.

The study presents two cases of orthodontic treatment and implant-prosthetic rehabilitation of maxillary hypodontia. In the first case, after obtaining space with fixed braces, a decision was made to insert a mini implant at the site of the missing upper right lateral incisor, on which a porcelain-fused-to-metal (PFM) crown was fixed. In the second case, after the expected effect of orthodontic treatment was achieved, the missing upper right lateral incisor and the missing upper right first bicuspid were restored with a PFM bridge, while the other missing upper left bicuspid was restored with a PFM bridge based on the implant. In the presented cases, effective orthodontic treatment was followed by an implant-prosthetic technique with the use of mini implants and fixed dentures, which allowed morphological, functional, and esthetic restoration of missing teeth. Both patients have had the restorations for 10 years and so far, have reported no functional or esthetic problems.

The applied orthodontic treatment and implant-prosthetic rehabilitation fulfilled the esthetic and functional needs of the patients. It must be emphasized that a treatment of an adult person with hypodontia may be disturbed by unforeseen circumstances, which may require corrections of the initial treatment plan.
\end{abstract}

KEY wORDS: hypodontia, fixed braces, mini implants, crown and bridges.

J Stoma 2018; 71, 4: 381-386

DOI: https://doi.org/10.5114/jos.2018.83413

\section{INTRODUCTION}

Hypodontia is defined as the congenital absence of tooth germs, which may be an isolated trait or part of a syndrome. It is defined as the absence of one to six tooth germs $[1,2]$. Abnormal number of teeth recognized after the period of physiological tooth eruption should be diagnosed as hypodontia. A metaanalysis of the prevalence of this pathology showed that the absence of at least one tooth may occur in $3.4 \%$ up to $10.1 \%$ of people [3]. The absence of lateral maxillary incisors occurs in $1.3-2.2 \%$ of cases and it is typically bilateral (59.5\%). Hypodontia is more frequent in females than males $[1,4,5]$, and commonly occurs in the family $[6,7]$. The risk of its occurrence among first-degree relatives of affected subjects is almost 15 -fold higher than in the families unaffected by this pathology [8].

\section{JOURNAL OF} STOMATOLOGY 
Patients with hypodontia suffer from numerous functional and esthetic problems. Therefore, their treatment must be complex and usually involves orthodontic, implantoprosthetic, logopedic, and psychological therapies [9]. Treatment of hypodontia should lead to restoration of missing teeth, proper occlusion and articulation as well as to improvement in esthetics, pronunciation, and mental status of the patient. The choice of therapeutic procedure depends on numerous factors, e.g., skeletal growth pattern, type of occlusal disturbance, deficit

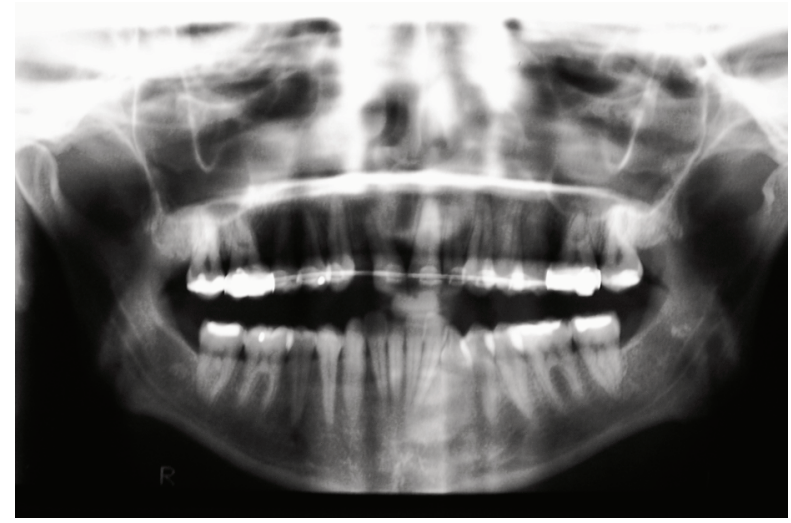

FIGURE 1. Case 1. Orthodontic treatment

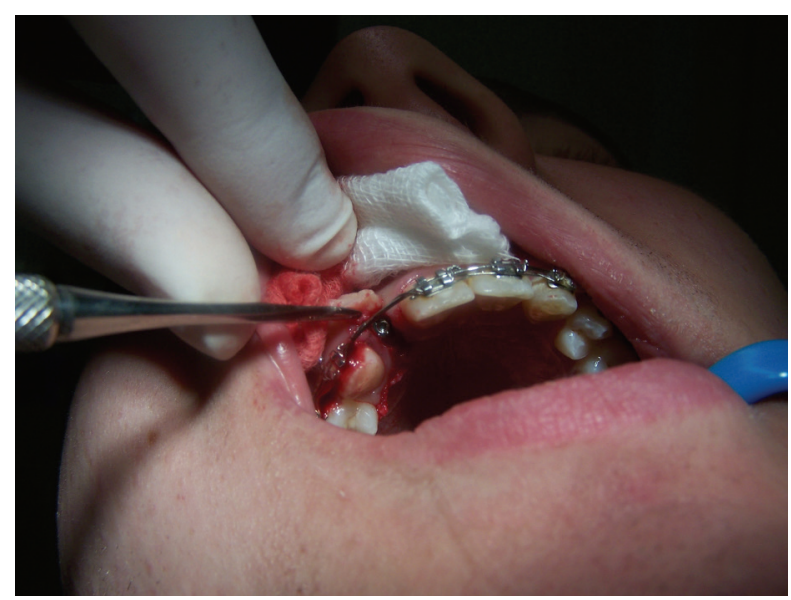

FIGURE 2. Case 1. Implantation in the region of missing tooth 12

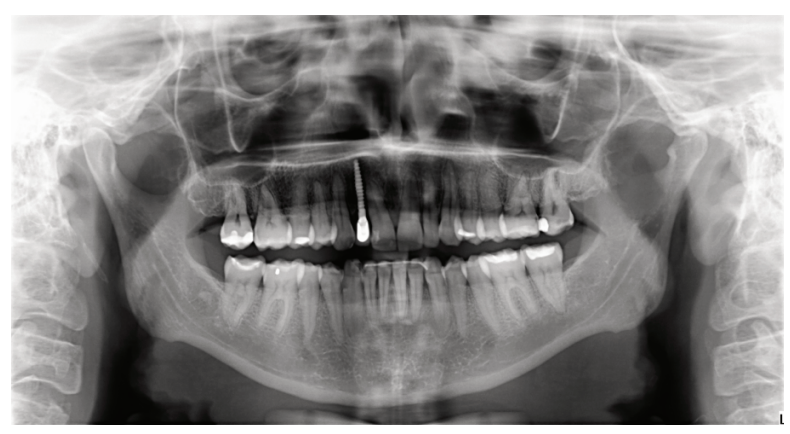

FIGURE 3. Case 1. Mini-implant inserted in the region of missing tooth 12 of bone density in the area of tooth germs absence, age, facial profile, arch width, and shape of canines.

Basic therapeutic procedures include orthodontic closure or opening of the space and implant-prosthetic rehabilitation $[5,7,10-13]$. The aim of the study is to present possible therapeutic procedures in the treatment of hypodontia of maxillary teeth in complex anatomical and biological conditions in adult patients.

\section{CASE REPORTS}

\section{CASE 1}

In the first case, hypodontia of the right lateral maxillary incisor was observed. Also, the upper right canine was located at the site of the lateral incisor and there was also diastema, midline shift, and crowding of teeth in the lower arch. Following the analysis of X-ray images (pantomographic and cephalometric), an assessment of diagnostic models and performance of the series of examinations of the alveolar ridge in the area of tooth 12 were completed and a treatment plan was presented, which included change of a canine tooth shape with a composite or restoration of a site for the upper right incisor with the use of fixed braces and restoration of this tooth on the implant. An alternative solution was to fabricate a bridge. After analyzing the possibility of significant loss of healthy tissues in pillars surrounding the space that might result from the fabrication of a bridge as well as the risk of failure and esthetic complications, the patient chose orthodontic treatment and implant-prosthetic restoration of the missing tooth.

In the first stage of treatment, fixed braces with bands were applied. In the course of treatment, the following tools were used: elastic traction for tooth 13 and 11, figure-eight ligature for tooth 11 and 21, expansion spring between tooth 13 and 11, and traction for distalization of tooth 13 (to restore space for tooth 12) (Figure 1). The shape of tooth 11 and 21 as well as inferior incisors and canines was corrected. Since the patient got pregnant during the orthodontic treatment, implant treatment was postponed. After removal of the fixed braces, a retainer was cemented in the mandible and a Maryland bridge was fabricated in the maxilla as a temporary restoration until pregnancy completion. An upper retention plate was also fabricated. Orthodontic treatment resulted in obtaining enough space for restoration of the right lateral maxillary incisor.

A few months after delivery, the patient reported for implant treatment. Check-up X-ray revealed a problem with a root angulation of tooth 11 and 13. The obtained space was $4.2 \mathrm{~mm}$ at the most critical site, what posed a serious problem for implantation. After informing the patient about the situation and discussing further treatment possibilities, a decision was made to insert a mini-implant with a diameter of $1.8 \mathrm{~mm}$ and $15 \mathrm{~mm}$ long (IMTEC) at 


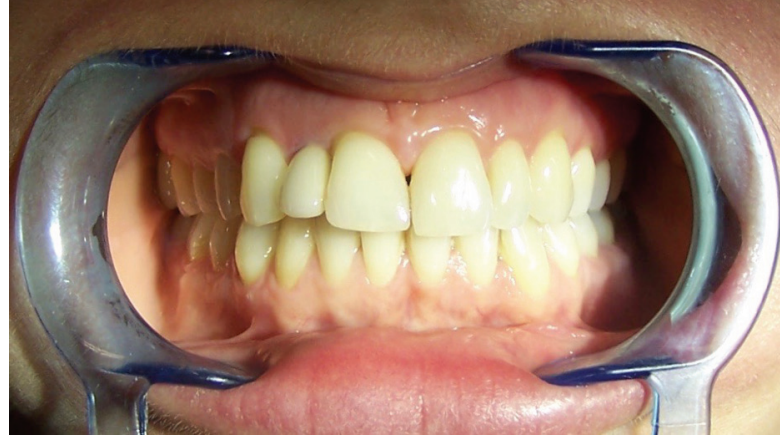

FIGURE 4. Case 1. Porcelain-fused-to-metal crown cemented on the mini-implant. The control photography after 10 years

the site of tooth 12 (Figures 2 and 3). The mini-implant was inserted transmucosally under local anesthesia. Primary stabilization was obtained at the level of $35 \mathrm{Ncm}$ (Figure 3). The implant was supplied with a temporary crown, which was excluded from the occlusion. After 6 months, a metal ceramic (porcelain-fused-to-metal - PFM) crown was cemented on the implant, which is still in use today. The restoration was checked every 12 months during clinical and radiological assessment. PFM crown cemented on the mini-implant. The control photography after 10 years (Figure 4 ).

\section{CASE 2}

The other case presented with hypodontia of teeth: $12,14,24,25$, and 36, upper arch spacing, large diastema, papillary attachment of the upper lip frenulum, small tooth 22 , and tooth 15 rotated $180^{\circ}$. Following the analysis of X-ray images (pantomographic and cephalometric) and assessment of diagnostic models, a decision was made to use fixed braces aimed at aligning the upper teeth, restoring a site for tooth 12 and 24, and closing the diastema. The planned procedures also included implantation in the area of tooth 12, 14, and 24 , and widening of tooth 22 with a composite. The patient was informed about the treatment plan and about an alveolar bone deficit, both horizontal and vertical, at the site of the missing tooth germs.

In the first stage of treatment, fixed braces - MMS low profile brackets, were used. Ligature was applied to teeth $11,21,13,15,21,22,23$, and 26 , active spring was applied on teeth 11 and 13, and a chain on teeth 11 and 23 (Figure 5). The orthodontic treatment resulted in the closure of diastema, correction of occlusal disturbances (derotation of tooth 15 to 14 and mesialization of tooth 23), and creating a room for implants at the site of missing teeth. After removal of braces, a retainer was cemented. Due to recurrent inflammation of the incisive papilla, a decision was made to surgically remove the frenulum of the upper lip under local anesthesia. Inflammation no longer occurred after the surgery.

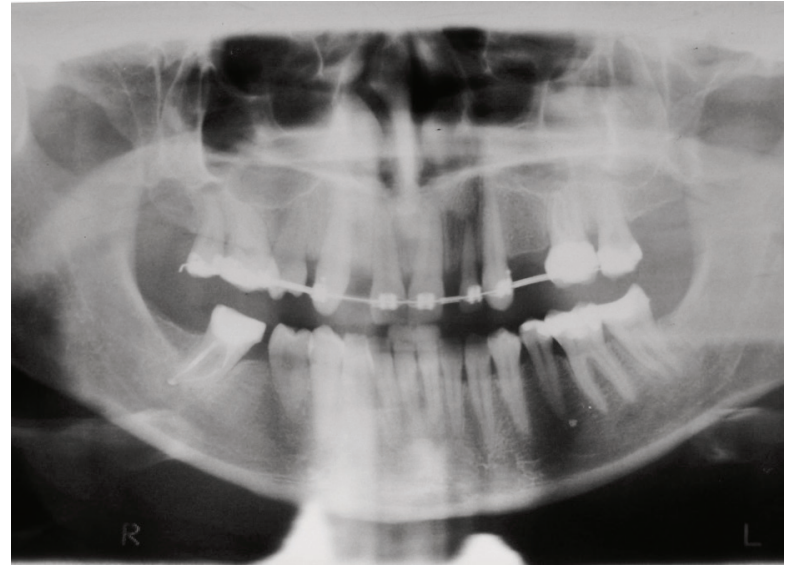

FIGURE 5. Case 2. Orthodontic treatment

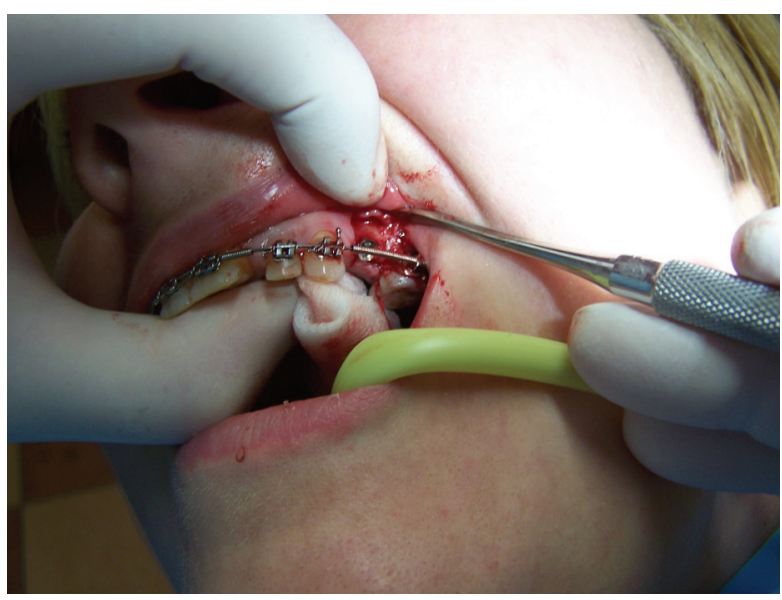

FIGURE 6. Case 2. Implantation in the region of missing tooth 24

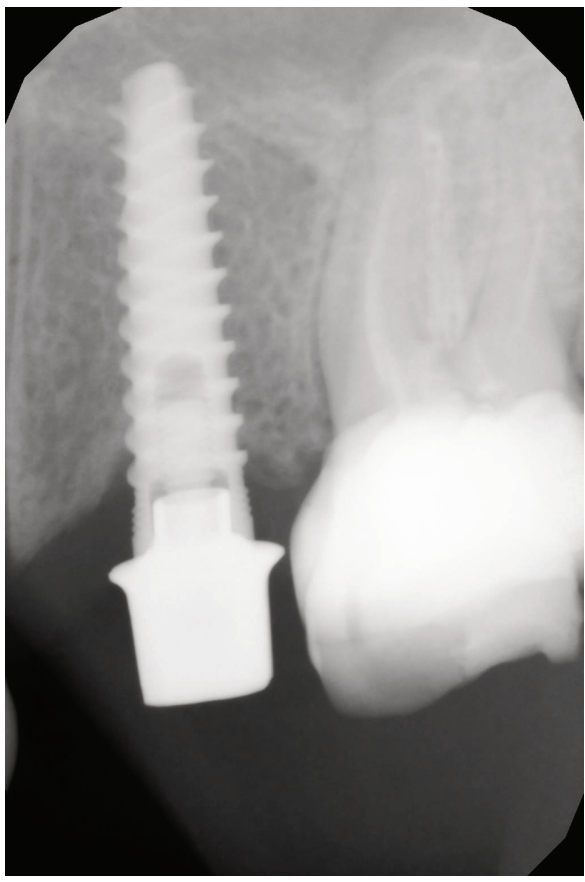

FIGURE 7. Case 2. Mini-implant inserted in the region of missing tooth 24 


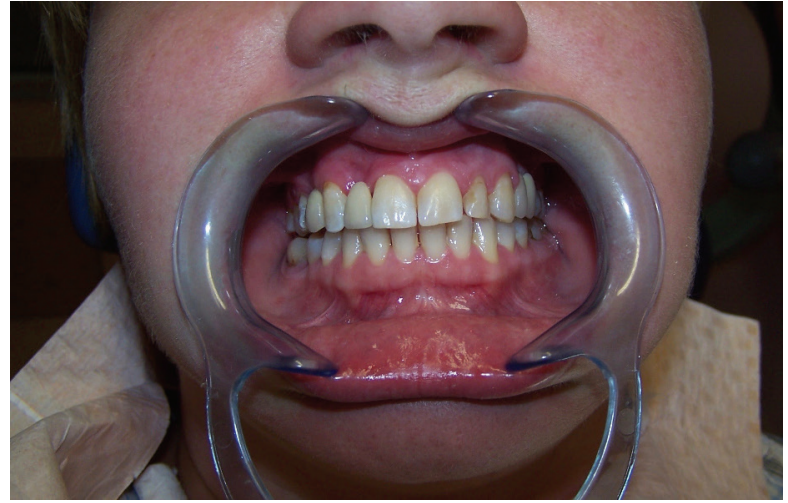

FIGURE 8. Case 2. Porcelain-fused-to-metal bridges in the area 12, 14, 24, and 25. The control photography after 10 years

The next stage of therapy was the insertion of three implants: one at the site of the upper right lateral incisor and two implants at the site of the first upper bicuspids, right and left. The implants were rejected twice in the area 12 and 14. In the area 24, the inserted MIS implant with a diameter of $3.75 \times 16$ was integrated with the bone (Figures 6 and 7). For this reason, metal ceramic (PFM) bridges were fabricated to restore the missing tooth $(12,14$, and 25) based on the implant in the area 24 . The PFM bridges were subject to a periodic clinical and radiological assessment. In the assessment, the following properties were included: retention, marginal adaptation and fitting, anatomical shape, color, structure of the veneering material, presence/absence of: staining, periapical changes, and recurrent caries. PFM bridges in the area $12,14,24$, and 25 . The control photography after 10 years (Figure 8 ).

\section{DISCUSSION}

Early diagnosis of agenesis, proper management of the patient, and fast multi-specialist treatment may protect the patient against irreversible consequences of hypodontia. Planning a therapy should be based on a radiological diagnosis and analysis of diagnostic models.

Restoration of the upper lateral incisor with a mini implant and ceramic crown is a good solution with a long-term prognosis. It does not require stripping of teeth surrounding the space and it restores integrity of the dental arch. Moreover, the patient is not exposed to an expensive, painful, and long-term treatment with augmentation techniques. Currently, this is the best method to restore a single tooth. Long-term studies confirm predictability, functionality, and durability of implant reconstructions [14-18].

The use of a narrow implant and little-invasive technique of transmucosal implantation is also an alternative to fixed restorations, such as bridges. The use of narrow implants to restore single missing teeth brings a satisfactory and long-term clinical and esthetic effect. According to Polizzi et al. [18], the success of this method in the period of 3 to 7 years amounts to $96.7 \%$. Many authors who use narrow implants have also conducted prospective studies. Romeo et al. [14] observed clinical success of $96.1 \%$ in the maxilla and $96.9 \%$ in the mandible after 7 years of follow-up of implants with a narrow diameter. Similar results were obtained by Comfort et al. [16] (96\%), Zinsli et al. [15] (98.7\% after 5 years and $96.6 \%$ after 6 years of follow-up), and Renouard and Nisand [19] (95.5\% after 10 years of follow-up). The use of narrow implants is not a new method. In the 70's of the previous century, Dino Garbaccio used bicortical screws, which are still successfully used by many implantologists. On the basis of many years of observation, it was found that monophasic implants provide the patient with a little invasive implantation procedure and an immediate esthetic effect - the patient is managed with a temporary crown $[14,19,20]$.

The selection of patients for such a therapy is based on their profile, type of occlusal disturbance, height of the lip line, and their personal expectations. Different implantation systems allow a recognition of various anatomical limitations, such as low bone density or insufficient space in the dental arch. There must always be an alternative treatment plan in the form of the conventional prosthetic restorations or the canine mesialization and correction of a canine shape with a porcelain or composite veneer [21], e.g., in case of biological or financial problems occurrence.

In the presented cases, effective orthodontic treatment was followed by an implant-prosthetic technique with the use of mini implants and fixed dentures, which allowed morphological, functional, and esthetic restoration of a missing teeth. Both patients have had the restorations for 10 years and so far, have reported no functional or esthetic problems.

\section{CONCLUSIONS}

The applied orthodontic treatment and implantprosthetic rehabilitation fulfilled the esthetic and functional needs of the patients. It must be emphasized that treatment of an adult person with hypodontia may be disturbed by unforeseen circumstances, which may require corrections of the initial treatment plan.

\section{CONFLICT OF INTEREST}

The authors declare no potential conflicts of interest with respect to the research, authorship, and/or publication of this article. 


\section{References}

1. Fekonja A. Hypodontia in orthodontically treated children. Eur J Orthod 2005; 27: 457-460.

2. De Coster PJ, Marks LA, Martens LC, Huysseune A. Dental agenesis: genetic and clinical perspectives. J Oral Pathol Med 2009; 38: 1-17.

3. Polder BJ, Van't Hof MA, Van der Linden FP, Kuijpers-Jagtman AM. A meta-analysis of the prevalence of dental agenesis of permanent teeth. Community Dent Oral Epidemiol 2004; 32: 217-226.

4. Robertsson S, Mohlin B. The congenitally missing upper lateral incisor. A retrospective study of orthodontic space closure versus restorative treatment. Eur J Orthod 2000; 22: 697-710.

5. Pinho T, Maciel P, Pollmann C. Developmental disturbances associated with agenesis of the permanent maxillary lateral incisor. British Dent J 2009; 207: E25.

6. Scarel RM, Trevilatto PC, Di Hipólito Jr O, et al. Absence of mutations in the homeodomain of the MSX1 gene in patients with hypodontia. Am J Med Genet 2000; 92: 346-349.

7. Näsman M, Forsberg CM, Dahllöf G. Long-term dental development in children after treatment for malignant disease. Eur J Orthod 1997; 19: 151-159.

8. Pinho T, Pollmann C, Calheiros-Lobo MJ, et al. Craniofacial repercussions in maxillary lateral incisors agenesis. Int Orthod 2011; 9: 274-285.

9. Closs LQ, Reston EG, Tessarollo F, et al. Multidisciplinary approach in the rehabilitation of missing lateral incisors: a new trend in daily practice. Oper Dent 2012; 37: 458-463.

10. Akkaya N, Kiremitc A, Kansu O. Treatment of a patient with oligodontia: a case report. J Contemporary Dent Practice 2008; 9: 121-127.

11. Silveira GS, Mucha JN. Agenesis of maxillary lateral incisors: treatment involves much more than just canine guidance. Open Dent J 2016; 10: 19-27.

12. Carter NE, Gillgrass TJ, Hobson RS, et al. The interdisciplinary management of hypodontia: orthodontics. British Dent J 2003; 194: 361-366.

13. Tanaka O, Kreia TB, Maciel JVB, Camargo ES. The agenesis of maxillary lateral incisors: closing or reopening the space? Dent Press J Orthod 2003; 2: 27-35.

14. Romeo E, Lops D, Amorni L, et al. Clinical and radiographic evaluation of small-diameter $(3.3-\mathrm{mm})$ implants followed for 1-7 years: a longitudinal study. Clin Oral Implants Res 2006; 17: 139-148.

15. Zinsli B, Sagesser T, Mericske E, Mericske-Stern R. Clinical evaluation of small-diameter iti implants: a prospective study. Int J Oral Maxillofac Implants 2004; 19: 92-99.

16. Comfort MB, Chu FC, Chai J, et al. A 5-year prospective study on small diameter screw-shaped oral implants. J Oral Rehabil 2005; 32: 341-345.

17. King P, Maiorana C, Luthardt RG, et al. Clinical and radiographic evaluation of a small-diameter dental implant used for the restoration of patients with permanent tooth agenesis (hypodontia) in the maxillary lateral incisor and mandibular incisor regions: a 36-month follow-up. Int J Prosthodont 2016; 29: 147-153.

18. Polizzi G, Fabbro S, Forri M, et al. Clinical application of narrow Branemark System implants for single-tooth restoration. Int J Oral Maxillofac Implants 1999; 14: 496-503.

19. Renouard F, Nisand D. Impact of implant length and diameter on survival rates. Clin Oral Implants Res 2006; 17 Suppl 2: 35-51.

20. Garbaccio D. The Garbaccio bicortical self-threading screw. Riv Odontostomatol Implantoprotesi 1983; 1: 53-56.

21. Filius MA, Cune MS, Raghoebar GM, et al. Prosthetic treatment outcome in patients with severe hypodontia: a systematic review. J Oral Rehabil 2016; 43: 373-387. 
